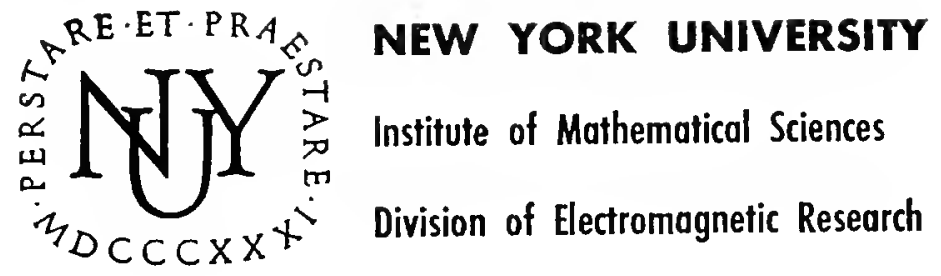

RESEARCH REPORT No.BR-36

\title{
Asymptotic Estimates for the Sturm-Liouville Spectrum
}

\author{
HARRY HOCHSTADT
}

Air Force Office of Scientific Research

Contract No. AF 49(638)-229

Project No. 47500

FE BR U ARY, 1961 
NEW YORK UNIVERSITY

Institute of Mathematical Sciences

Division of Electromagnetic Research

Research Report No. BR-36

ASYMPTOTIC ESTIMATES FOR THE STURM-ILTOUVIIJE SPECTRUM

Herry Hochstadt

Hary Hochstadt

Marri Yline

Morris Kline

Director

February, 1961

Qualified requestors may obtain copies of this report from the ASTIA Document Service Center, Arlington Hall Station, Arlington 13, Virginia. Department of Defense contractors must be established for ASIIA services, or have their 'need to know' certified by the cogntzant military agency of their project or contract. 

Abstract

It is shown that the differential equation

$$
y^{\prime \prime}+[\lambda+\Phi(x)] y=0
$$

can, under suftable conditions, be solved by assuming a solution of the form

$$
y=A(x) \sin \phi(x)
$$

where

$$
\begin{aligned}
& \varphi^{\prime}(\mathrm{x})=\sqrt{\lambda+\Phi(\mathrm{x})}+\frac{1}{4} \frac{\Phi^{\prime}(\mathrm{x})}{\lambda+\Phi(\mathrm{x})} \sin 2 \varphi(\mathrm{x}) \\
& \mathrm{A}^{\prime}(\mathrm{x})=-\mathrm{A}(\mathrm{x}) \frac{\Phi^{\prime}(\mathrm{x})}{2 \lambda+2 \Phi(\mathrm{x})} \cos ^{2} \varphi(\mathrm{x}) .
\end{aligned}
$$

Use of the first equation leads, when boundary conditions are applied, to asymptotic estimates of the elgenvalues.

In particular, in the case of Hill's equation, it is shown that the instablity intervals vanish faster than any inverse power of $\mathrm{k}, \mathrm{k}$ belng the order of the corresponding elgenvalues, when $\Phi(x)$ is an analytic function. 
Table of Contents

Introduction

1

The method

2

The Sturm-Liouville problem

3

Hill's equation

14

The stablity intervals for HilI's equation

18

A result due to Erdélyı

21

A special case

23

Bibliography

26 


\section{Introduction}

In the classical Sturm-Liouville problem one is concerned with the differential equation

$$
\frac{d}{d x} p(x) \frac{d y}{d x}+(\lambda \rho(x)+g(x)) y=0
$$

subject to the boundary conditions

$$
\begin{aligned}
& \alpha y(0)+\beta y^{\prime}(0)=0 \\
& \gamma y(\pi)+\delta y^{\prime}(\pi)=0 .
\end{aligned}
$$

The fincte interval under consideration can be taken to be the interval $(0, \pi), \lambda$ is a parameter and $p(x), \rho(x), g(x)$ are real functions, and furthermore $p(x)$ and $\rho(x)$ are positive. $\alpha, \beta, \gamma, \delta$ are constants such that $|\alpha|+|\beta| \neq 0$ and $|\gamma|+|\delta| \neq 0$. One is then interested in determining those values of $\lambda$ for which non-trivial solutions of the differential equation can be found, which satisfy the boundary conditions.

If $p(x)$ and $\rho(x)$ are doubly differentiable, one can by means of the Llouville transformation reduce the equation to the form

$$
\frac{d^{2} y}{d x^{2}}+(\lambda+\Phi(x)) y=0 .
$$

Borg $[I]$ discussed this equation and the asymptotic form of the characteristic values of $\lambda$. His chief interest was a discussion 
of the inverse Sturm-Liouvilie problem, where one is interested in reconstructing the differential equation from a knowledge of the Sturm-If ouvilie spectrum.

In this publication a method is developed, which leads to Borg's results in a very efficient fashion, and has the additional advantage of ylelding 1mproved error estimates. Furthermore under falrly general conditions one can easily obtain asymptotic series for large eigenvalues and the corresponding eigenfunctions. The method leads to particularly precise information in the case of Hill's equation.

As will be observed subsequently the method is intimately related to the $\mathrm{W}-\mathrm{K}-\mathrm{B}$ method, with the additional advantage of providing a rigorous foundation for the latter method.

The method

If we consider the differential equation

$$
y^{\prime \prime}+Q^{2}(x) y=0
$$

where $Q(x)$ is a differentiable non-vanishing function, it seems reasonable to assume a solution of the form

$$
y=A(x) \sin p(x) \cdot
$$

If in addition one postulates that

$$
y^{\prime}=A(x) G(x) \cos p(x),
$$


one can by means of the original equation show by a direct calculation that

$$
\begin{aligned}
& \varphi^{\prime}(x)=Q(x)+\frac{1}{2} \frac{Q^{\prime}(x)}{Q(x)} \sin 2 \varphi(x) \\
& A^{\prime}(x)=-A(x) \frac{Q^{\prime}(x)}{Q(x)} \cos ^{2} \varphi(x) .
\end{aligned}
$$

Similarly, if one assumes that

$$
\begin{aligned}
& y=A(x) \cos \phi(x) \\
& y^{\prime}=-A(x) \quad Q(x) \sin p(x)
\end{aligned}
$$

one obtains

$$
\begin{aligned}
& P^{\prime}(x)=Q(x)-\frac{1}{2} \frac{Q^{\prime}(x)}{Q(x)} \sin 2 p(x) \\
& A^{\prime}(x)=-A(x) \frac{Q^{\prime}(x)}{Q(x)} \sin ^{2} p(x) .
\end{aligned}
$$

Evidently once $p(x)$ has been found from the flrst of these equations, $A(x)$ can be obtained by an integration from the second equation, and it also follows that $A(x) / A(0)$ is a positive function, since it is an exponential.

The Sturm-Liouville problem

We now turn to the equation

$$
y^{\prime \prime}+[\lambda+\Phi(x)] y=0
$$




$$
\begin{aligned}
& -4- \\
& \alpha y(0)+\beta y^{\prime}(0)=0 \\
& \gamma y(\pi)+\delta y^{\prime}(\pi)=0,
\end{aligned}
$$

and subdivide the problem into four distinct subcases:

Ia.

$$
\begin{aligned}
& \alpha=\gamma=1 \\
& \beta=\delta=0
\end{aligned}
$$

IIa.

$$
\begin{aligned}
& \alpha=\delta=1 \\
& \beta=0
\end{aligned}
$$

$$
\gamma \text { arbitrary }
$$

Ib.

$$
\begin{aligned}
& \beta=\delta=1 \\
& \alpha, \gamma \text { arbitrary }
\end{aligned}
$$

IIb.

$$
\begin{aligned}
& \beta=\gamma=1 \\
& \delta=0 \\
& \alpha \text { arbitrary }
\end{aligned}
$$

Problem Ia.

We now consider

$$
y^{\prime \prime}+[\lambda+\Phi(x)] y=0
$$

where $y(0)=y(\pi)=0$,

and $\Phi(x)$ is a differentiable function of mean value zero, i.e.

$$
\int_{0}^{\pi} \Phi(x) d x=0 .
$$


Under the assumption that

$$
\begin{aligned}
y & =A(x) \sin \varphi(x) \\
y^{\prime} & =A(x) \sqrt{\lambda+\Phi(x)} \cos \varphi(x)
\end{aligned}
$$

we obtain

$$
\varphi^{\prime}(x)=\sqrt{\lambda+\Phi(x)}+\frac{1}{4} \frac{\Phi^{\prime}(x)}{\lambda+\Phi(x)} \sin 2 \varphi(x)
$$

where

$$
\begin{aligned}
& p(0)=0 \\
& p(\pi)=\mathrm{k} \pi
\end{aligned}
$$

and $\mathrm{k}$ is any sufficiently large integer. Here sufficiently large means so large that for the corresponding value of $\lambda$

$$
\lambda+f(x)>0
$$

The differential equation can be rewritten as an integral equation

$$
p(x)=\int_{0}^{x} \sqrt{\lambda+\Phi(x)} d x+\frac{1}{4} \int_{0}^{x} \frac{\Phi(x)}{\lambda+\Phi(x)} \sin 2 \varphi(x) d x .
$$

From this equation se see that

$$
i(x)=\int_{0}^{x} \sqrt{\lambda+2(x)} a x+o\left(\frac{1}{\lambda}\right)
$$


and by an 1teration

$$
\begin{gathered}
\varphi(x)=\int_{0}^{x} \sqrt{\lambda+\Phi(x)} d x+\frac{1}{4} \int_{0}^{x} \frac{\Phi^{\prime}(x)}{\lambda+\Phi} \sin \left(2 \int_{0}^{x} \sqrt{\lambda+\Phi(x)} d x\right) d x \\
+\frac{\left(\frac{1}{\lambda^{2}}\right) .}{}
\end{gathered}
$$

We can get estimates for $\lambda$ for large values of $k$ from the boundary condition

$$
\begin{gathered}
\int_{0}^{\pi} \sqrt{\lambda+\Phi(x)} d x+\frac{1}{4} \int_{0}^{\pi} \frac{\Phi^{\prime}(x)}{\lambda+\Phi(x)} \sin \left(2 \int_{0}^{x} \sqrt{\lambda+\Phi(x)} d x\right) d x \\
+o\left(\frac{1}{\lambda^{2}}\right)=k \pi .
\end{gathered}
$$

If $\Phi^{\prime}(x)$ is differentiable an integration by parts can be applied to the second integral above and one then finds that

$$
\sqrt{\lambda}=k+\frac{c_{1}}{8 k^{3}}+o\left(\frac{1}{k^{4}}\right) \text {. }
$$

where

$$
c_{1}=\frac{1}{\pi} \int_{0}^{\pi} \Phi^{2}(x) d x+\frac{\Phi^{\prime}(\pi)-\Phi^{\prime}(0)}{\pi},
$$

or

$$
\lambda=k^{2}+\frac{c_{1}}{4 k^{2}}+o\left(\frac{1}{k^{3}}\right) .
$$


If, on the other hand, $\Phi^{\prime}(x)$ is not differentiable we expand $\Phi(x)$ in a Fourier serles by continuing $\Phi(x)$ outside its interval of definftion $(0, \pi)$ as an even function of period $2 \pi$ and mean value zero. Then

$$
\begin{aligned}
\Phi(x) & =\sum_{1}^{\infty} a_{n} \cos n x, \\
a_{n} & =\frac{o\left(\frac{1}{n^{2}}\right)}{}
\end{aligned}
$$

and we find that

$$
\sqrt{\lambda}=k+\frac{a}{4 k}+\frac{1}{8 \pi k^{3}} \int_{0}^{\pi} \Phi^{2}(x) d x+0\left(\frac{1}{k^{4}}\right) .
$$

Now that an asymptotic estimate of $\lambda$ is known one can find asymptotic estimates of $\phi(x)$ and $A(x)$ and therefore $y(x)$. Then one finds that

$$
y(x)=\frac{A(0) \sin \left[2 k x+\frac{1}{k} \int_{0}^{x} \Phi(x) d x\right]}{[k+\Phi(x)]^{1 / 4}}\left[1+o\left(\frac{1}{k^{2}}\right)\right] .
$$

It 1 s interesting to compare the elgenvalues with those of the corresponding problem for $\Phi(x) \equiv 0$. Then

$$
y^{\prime \prime}+x y=0
$$




$$
\begin{aligned}
& y(0)=y(\pi)=0 \\
& \sqrt{x}=k
\end{aligned}
$$

and

$$
\sqrt{\lambda}-\sqrt{x}=\frac{c_{1}}{8 k^{3}}+0\left(\frac{1}{k^{4}}\right)
$$

for $\Phi^{\prime}(x)$ differentiable. When $\Phi^{\prime}(x)$ is not differentiable

$$
\sqrt{\lambda}-\sqrt{x}=\frac{a_{2 k}}{4 k}+\frac{1}{8 \pi k^{3}} \int_{0}^{\pi} \Phi^{2}(x) d x+0\left(\frac{1}{k}\right) .
$$

\section{Problem Ib.}

For the equation with constant coefficlents we have in this case

$$
\begin{aligned}
& y^{\prime \prime}+x y=0 \\
& c y(0)+y^{\prime}(0)=0 \\
& \gamma y(\pi)+y^{\prime}(\pi)=0
\end{aligned}
$$

so that

$$
\tan \sqrt{x} \pi=\frac{(\gamma-\alpha) \sqrt{x}}{x+\gamma \alpha} .
$$


One can show from this transcendental equation that

$$
\sqrt{x}=k+\frac{\gamma-\alpha}{\pi k}-\frac{\left(\frac{\gamma-\alpha}{\pi}\right)^{2}+\frac{1}{3} \frac{\gamma^{3}-\alpha^{3}}{\pi}}{k^{3}}+o\left(\frac{1}{k^{4}}\right) .
$$

When we turn to the more general problem

$$
\begin{aligned}
& y^{\prime \prime}+[\lambda+\Phi(x)] y=0 \\
& \alpha y(0)+y^{\prime}(0)=0 \\
& \gamma(\pi)+y(\pi)=0
\end{aligned}
$$

we assume a solution of the form

$$
\begin{aligned}
y & =A(x) \sin (\Phi(x)+\Delta) \\
y^{\prime} & =A(x) \sqrt{\lambda+\Phi(x)} \cos (\varphi(x)+\Delta) .
\end{aligned}
$$

If

$$
\Delta=-\tan ^{-1} \frac{\sqrt{\lambda+\Phi(0)}}{\alpha}
$$

the boundary condition at $x=0$ is satisfied. As before we find

$$
\varphi^{\prime}(x)=\sqrt{\lambda+\Phi(x)}+\frac{1}{4} \frac{\Phi^{3}(x)}{\lambda+\Phi(x)} \sin [2 \varphi+2 \Delta]
$$


with the boundary conditions

$$
\begin{aligned}
& \varphi(0)=0 \\
& \varphi(\pi)=k \pi+\tan ^{-1} \frac{\sqrt{\lambda+\Phi(0)}}{\alpha}-\tan ^{-1} \frac{\sqrt{\lambda+\Phi(\pi)}}{\gamma} .
\end{aligned}
$$

As in Problem Ia. we can rewrite this equation as an integral equation and obtain the asymptotic solution

$$
\begin{aligned}
\varphi(x)= & \int_{0}^{x} \sqrt{\lambda+\Phi(x)} d x+\frac{1}{4} \int_{0}^{x} \frac{\Phi^{\prime}(x)}{\lambda+\Phi(x)} \cdot \\
& \sin \left[2 \int_{0}^{x} \sqrt{\lambda+\Phi(x)} d x+2 \Delta\right] d x+0\left(\frac{1}{\lambda^{2}}\right) .
\end{aligned}
$$

Applying the boundary condition at $x=\pi$ we find

$$
\begin{aligned}
\int_{0}^{\pi} \sqrt{\lambda+\Phi(x)} d x+\frac{1}{4} \int_{0}^{\pi} \frac{\Phi(x)}{\lambda+\Phi(x)} \sin \left[2 \int_{0}^{x} \sqrt{\lambda+\Phi(x)} d x+2 \Delta\right] d x \\
+0\left(\frac{1}{\lambda}\right)=k \pi+\tan ^{-1} \frac{\sqrt{\lambda+\Phi(0)}}{\alpha}-\tan ^{-1} \frac{\sqrt{\lambda+\Phi(\pi)}}{\gamma} .
\end{aligned}
$$

For $\Phi^{\prime}(\mathrm{x})$ differentiable we can solve this equation asymptotically and find 


$$
\begin{aligned}
\sqrt{\lambda}= & +\frac{\gamma-\alpha}{\pi k}-\frac{\left(\frac{\gamma-\alpha}{\pi}\right)^{2}+\frac{1}{3} \frac{\gamma^{3}-\alpha^{3}}{\pi}}{k^{3}} \\
& +\frac{c_{3}}{8 k^{3}}+o\left(\frac{1}{k^{4}}\right)
\end{aligned}
$$

where

$$
c_{3}=\frac{1}{\pi}\left[\int_{0}^{\pi} \Phi^{2}(x) d x-\Phi^{2}(\pi)+\Phi^{2}(0)+4 \alpha \Phi(0)-4 \gamma \Phi(\pi)\right] .
$$

A comparison with the corresponding value of $\sqrt{ }$ shows that

$$
\sqrt{\lambda}-\sqrt{x}=\frac{c_{3}}{8 k^{3}}+o\left(\frac{1}{k^{4}}\right)
$$

When $\Phi^{\prime}(x)$ is not differentiable we proceed as before and expand $\Phi(x)$ in a Fourier series. We then obtain

$$
\sqrt{\lambda}-\sqrt{x}=\frac{\int_{0}^{\pi} \Phi^{2}(x) d x}{8 k^{3} \pi}-\frac{a_{2 k}}{4 k}+0\left(\frac{1}{k^{4}}\right) .
$$


Problem IIa.

The same methods apply to this problem. The results merely will therefore be stated.

The equation

$$
\begin{array}{r}
y^{\prime \prime}+x y=0 \\
y(0)=0 \\
\gamma y(\pi)+y^{i}(\pi)=0
\end{array}
$$

has the elgenvalues governed by

$$
\tan \sqrt{x} \pi=\frac{-\sqrt{x}}{\gamma}
$$

from which one can find that

$$
\sqrt{x}=k+\frac{1}{2}+\frac{\gamma}{\pi k}-\frac{y}{2 \pi k^{2}}+\frac{\frac{\gamma}{4}-\frac{y^{2}}{\pi}-\frac{1}{3} \gamma^{3}}{\pi k^{3}}+o\left(\frac{1}{k}\right) .
$$

For the equation

$$
\begin{array}{r}
y^{\prime \prime}+[\lambda+\Phi(x)] y=0 \\
y(0)=0 \\
\gamma y(\pi)+y^{2}(\pi)=0
\end{array}
$$


we have

$$
\begin{aligned}
& \varphi^{\prime}(\mathrm{x})=\sqrt{\lambda+\Phi(\mathrm{x})}+\frac{1}{4} \frac{\Phi^{\prime}(\mathrm{x})}{\lambda+\Phi(\mathrm{x})} \sin 2 \varphi(\mathrm{x}) \\
& \varphi(0)=0 \\
& \varphi(\pi)=k+1-\tan ^{-1} \frac{\sqrt{\lambda+\Phi(\pi)}}{\gamma} .
\end{aligned}
$$

We then find, for $\Phi^{\prime}(x)$ dffferentiable, that

$$
\sqrt{\lambda}-\sqrt{x}=\frac{1}{8 k^{3}} c_{2}+o\left(\frac{1}{k^{4}}\right)
$$

where

$$
c_{2}=\frac{I}{\pi}\left\{\int_{0}^{\pi} \Phi^{2}(x) d x-4 \gamma^{\Phi}(\pi)-\Phi^{\prime}(0)-\Phi^{\prime}(\pi)\right\} .
$$

When $\Phi^{\prime}(x)$ is not differentiable

$$
\sqrt{\lambda}-\sqrt{x}=\frac{\int_{0}^{\#} \Phi^{2}(x) d x}{8 k^{3}}+\frac{a_{2 k+1}}{4\left(k+\frac{1}{2}\right)}+0\left(\frac{1}{k^{4}}\right)
$$

\section{Problem ITb.}

Problem ITb can be solved Immediately by realfzing that under the transformation 


$$
\begin{aligned}
& x \rightarrow \pi-x \\
& \gamma \rightarrow-\alpha
\end{aligned}
$$

Problem IIa goes over into IIb and one then finds for $\Phi^{\prime}(x)$ differentiable

$$
\sqrt{\lambda}-\sqrt{x}=\frac{1}{8 k^{3}} c_{4}+o\left(\frac{1}{k^{4}}\right)
$$

where $c_{4}=\frac{1}{\pi}\left\{\int_{0}^{\pi} \Phi^{2}(x) d x+4 a \Phi(0)+\Phi^{\prime}(0)+\Phi^{\prime}(\pi)\right\}$

and for $\Phi^{\prime}(x)$ not differentiable

$$
\sqrt{\lambda}-\sqrt{x}=\frac{\int_{0}^{\pi} \Phi^{2}(x) d x}{8 k^{3} \pi}-\frac{a_{2 k+1}}{4\left(k+\frac{1}{2}\right)}+o\left(\frac{1}{k^{4}}\right) .
$$

\section{Hill's equation}

We now consider the case where $\Phi(x)$ is an even differentiable function of period $\pi$. For boundary conditions we use the following four possible sets [see reference 2].
1.) $y^{\prime}(0)=y^{\prime}\left(\frac{\pi}{2}\right)=0$
2.) $y(0)=y\left(\frac{\pi}{2}\right)=0$
3.) $y^{:}(0)=y\left(\frac{\pi}{2}\right)=0$
4.) $y(0)=y^{\prime}\left(\frac{\pi}{2}\right)=0$ 
which lead to solutions of the differential equation with the following character respectively

1.) even of period $\pi$

2.) odd of period $\pi$

3.) even of period $2 \pi$

4.) odd of period $2 \pi$.

These four cases correspond to Problems Ib, Ia, IIb, II considered in the previous section, respectively. There is a slight change in the sense that the interval under consideration now is the interval $\left(0, \frac{\pi}{2}\right)$, whereas before 1 was the interval $(0, \pi)$. The only change that must be made in cases 1.) and 2.) is that

$$
\varphi\left(\frac{\pi}{2}\right)=\mathrm{k} \pi
$$

We then find for cases 1.) and 2.)

$$
\sqrt{\lambda}=2 k+\frac{1}{64 k^{3} \pi} \int_{0}^{\pi} \Phi^{2}(x) d x+o\left(\frac{1}{k^{4}}\right) .
$$

In cases 3.) and 4.) we have to modify the boundary conaition at $\pi$ to

$$
\varphi\left(\frac{\pi}{2}\right)=(2 k+1) \frac{\pi}{2} .
$$


Then we find

$$
\sqrt{\lambda}=2 k+1+\frac{1}{64 k^{3} \pi} \int_{0}^{\pi} \Phi^{2}(x) d x+o\left(\frac{1}{k^{4}}\right) .
$$

In case 2.), for example, we can show easily that

$$
\varphi(\mathrm{x})=2 k \mathrm{x}+\psi(\mathrm{x})
$$

where $\psi(x)$ is an odd function of period $\pi$. If we use this form of $\varphi(\mathrm{x})$ as a trial solution in the equation

$$
\begin{aligned}
& \varphi^{\prime}(\mathrm{x})=\sqrt{\lambda+\Phi(\mathrm{x})}+\frac{1}{4} \frac{\Phi^{3}(\mathrm{x})}{\lambda+\Phi(\mathrm{x})} \sin 2 \varphi(\mathrm{x}) \\
& \varphi(0)=0 \\
& \varphi\left(\frac{\pi}{2}\right)=\mathrm{k} \pi
\end{aligned}
$$

we obtain

$$
\begin{aligned}
& \psi^{\prime}(x)=\sqrt{\lambda+\Phi(x)}-2 k+\frac{1}{4} \frac{\Phi^{\prime}(x)}{\lambda+\Phi(x)} \sin [4 k x+2 \psi(x)] \\
& \psi(0)=\psi\left(\frac{\pi}{2}\right)=0 .
\end{aligned}
$$

From this equation it is evident that $\psi(x)$ is an odd function, and the equation is invariant under the transformation

$$
x \rightarrow x+\pi
$$


Considering the boundary conditions on $\psi(x)$, the result is evident. We can also show that the function $A(x)$ has period $\pi$. The differential equation for $A(x)$ is

$$
\begin{aligned}
& A^{\prime}(x)=-A(x) \frac{\Phi^{\prime}(x)}{2(\lambda+\Phi(x))} \cos ^{2} \varphi(x) \\
& \frac{A^{\prime}(x)}{A(x)}=-\frac{\Phi^{2}(x)}{4(\lambda+\Phi(x))}[1+\cos (4 k x+2 \psi(x))] .
\end{aligned}
$$

The equation is invariant under the transformation

$$
x \rightarrow x+\pi \text {. }
$$

Furthermore

$$
\begin{array}{r}
\ln \frac{A(\pi)}{A(0)}=-\frac{1}{4} \int_{0}^{\pi} \frac{\Phi^{\prime}(x)}{\lambda+\Phi(x)}[1+\cos 4 k x \cos 2 \psi(x) \\
-\sin 4 k x \sin 2 \psi(x)] d x
\end{array}
$$

where the first integral vanishes by an immediate integration and the second and third terms vanish because they represent cosine and sine Fourier coefficients of odd and even functions. Thus

$$
\ln \frac{A(\pi)}{A(0)}=0
$$


so that $A(\pi)=A(0)$. Thus the solution

$$
y=A(x) \sin \varphi(x)
$$

for case 2.) can be seen to be an odd function of period $\pi$ directly. It is easily shown that

$$
\psi(x)=\frac{1}{4 k} \int_{0}^{x} \Phi(x) d x+o\left(\frac{1}{k^{2}}\right)
$$

and

$$
\ln \frac{A(x)}{A(0)}=1+0\left(\frac{1}{k^{2}}\right)
$$

so that

$$
y=A(0) \sin \left[4 k x+\frac{I}{4 k} \int_{0}^{x} \Phi(x) d x\right]+o\left(\frac{1}{k^{2}}\right) .
$$

Evidently these asymptotic estimates can be improved if necessary.

The stability intervals for Hill's equation

It is well known $[2]$ that if the eigenvalues corresponding to solutions of periodicity $\pi$ be denoted by $\lambda_{0}, \lambda_{I}, \lambda_{2}, \ldots$ and those of periodicity $2 \pi$ be denoted by $\lambda_{1}^{1}, \lambda_{2}^{1}, \lambda_{3}^{1}, \ldots$ they form a sequence ordered as follows

$$
\lambda_{0}<\lambda_{1}^{\prime} \leq \lambda_{2}^{\prime}<\lambda_{1} \leq \lambda_{2}<\lambda_{3}^{\prime} \leq \lambda_{4}^{\prime}<\lambda_{3} \leq \lambda_{4}<\cdots
$$

For all $\lambda$ in the intervals $\left(\lambda_{0}, \lambda_{1}^{1}\right),\left(\lambda_{2}^{1}, \lambda_{1}\right),\left(\lambda_{2}, \lambda_{3}^{1}\right),\left(\lambda_{4}^{1}, \lambda_{3}\right) \ldots$ 
the differential equation has only bounded solutions. For $\lambda$ outside these intervals one solution is unbounded. Therefore the intervals $\left(-\infty, \lambda_{0}\right),\left(\lambda_{1}^{1}, \lambda_{2}^{1}\right),\left(\lambda_{1}, \lambda_{2}\right),\left(\lambda_{3}^{1}, \lambda_{4}^{1}\right), \ldots$ are known as the instability intervals and they are known to become small for high eigenvalues, but little is known regarding their order of smallness. An immediate consequence of the asymptotic formulas developed in the previous section is that

$$
\sqrt{\lambda_{k+1}}-\sqrt{\lambda_{k}}=O\left(\frac{1}{k}\right)
$$

from which we see that

$$
\lambda_{k+1}-\lambda_{k}=O\left(\frac{1}{k^{3}}\right),
$$

and similarly,

$$
\lambda_{k+1}^{\prime}-\lambda_{k}^{\prime}=O\left(\frac{1}{k^{3}}\right)
$$

When the function $\Phi(x)$ is analytic one can give much more precise estimates for the instability intervals. For example, for case 1.) we have

$$
\varphi\left(\frac{\pi}{2}\right)=\int_{0}^{\pi / 2} \sqrt{\lambda+\Phi(x)} d x-\frac{1}{4} \int_{0}^{\pi / 2} \frac{\Phi^{\prime}(x)}{\lambda+\Phi(x)} \sin 2 \varphi(x) d x=k \pi
$$

and for case 2.) 


$$
\varphi\left(\frac{\pi}{2}\right)=\int_{0}^{\pi / 2} \sqrt{\lambda+\Phi(x)} d x+\frac{1}{4} \int_{0}^{\pi / 2} \frac{\Phi^{\prime}(x)}{\lambda+\Phi(x)} \sin 2 \varphi(x) d x=k \pi
$$

When $\Phi(x)$ is analytic so is $\Phi(x)$. Therefore one can apply integration by parts to the integral

$$
\begin{aligned}
& \int_{0}^{\pi / 2} \frac{\Phi^{\prime}(x)}{\lambda+\Phi(x)} \sin 2 \varphi(x) d x=\int_{0}^{\pi / 2} \cos 2 \varphi(x) \frac{d}{d x} \frac{1}{2 \varphi^{\prime}(x)} \frac{\Phi^{\prime}(x)}{\lambda+\Phi(x)} d x \\
= & \int_{0}^{\pi / 2} \sin 2 \varphi(x) \frac{d}{d x} \frac{1}{2 \varphi^{\prime}(x)} \frac{d}{d x} \frac{1}{2 \varphi^{\prime}(x)} \frac{\Phi^{\prime}(x)}{\lambda+\Phi(x)} d x=\ldots \\
= & \int_{0}^{\pi / 2} \sin 2 \varphi(x)\left(\frac{d}{d x} \frac{1}{2 \varphi^{\prime}(x)}\right) 4 N^{i} \frac{\Phi^{\prime}(x)}{\lambda+\Phi^{\prime}(x)} d x,
\end{aligned}
$$

where the integrated temns vanish by virtue of the periodicity properties of the integrand. Since

$$
\begin{aligned}
& \varphi(x)=o(\sqrt{\lambda})=o(k) \\
& \varphi^{\prime}(x)=o(\sqrt{\lambda})=o(k)
\end{aligned}
$$

it follows that

$$
\int_{0}^{\pi / 2} \frac{\Phi^{\prime}(x)}{\lambda+\Phi(x)} \sin 2 \varphi(x) d x=0\left(\frac{1}{\lambda^{2 \pi+1}}\right)=0\left(\frac{1}{x^{4 N+2}}\right)
$$


for all values of $N$. By taking the difference of the equations for cases 1.) and 2.) we see that

$$
\sqrt{\lambda_{k+1}}-\sqrt{\lambda_{k}}=0\left(\frac{1}{k^{4 N+2}}\right)
$$

or

$$
\lambda_{k+1}-\lambda_{k}=0\left(\frac{1}{k^{4 N+1}}\right)
$$

since $\mathbb{N}$ is arbitrary we can conclude that the instabllity internals vanish faster then any negative power of $k$.

A similar process shows that when $\Phi(\mathrm{x})$ is $\mathrm{m}$ times differentiable

$$
\lambda_{k+1}-\lambda_{k}=0\left(\frac{1}{k^{m+1}}\right) \text {. }
$$

A result due to Erdslyi.

Erdélyi derived [3] some estimates for small $\Phi(x)$. These follow very simply by this method. We write $\epsilon \Phi(x)$ instead of $\Phi(x)$ and assume that $\epsilon$ is small. Then for case 2.) we obtain

$$
\begin{aligned}
& \varphi^{\prime}(x)=\sqrt{\lambda+\epsilon \Phi(x)}+\frac{1}{4} \frac{\epsilon \Phi^{\prime}(x)}{\lambda+\epsilon \Phi(x)} \sin 2 \varphi(x) \\
& \varphi(0)=0 \\
& \varphi\left(\frac{\pi}{2}\right)=k \pi .
\end{aligned}
$$

We now try to find a perturbation series solution for $p(x)$ of the form form $\varphi(x)=2 k x+\epsilon \psi(x)+\cdots$

and for $\lambda$ we use

$$
\lambda=4 k^{2}+\epsilon \mu+\cdots \cdot
$$


Retaining only $\in$ terms we have immediately

$$
\psi^{\prime}(x)=\frac{\mu+\Phi(x)}{4 k}+\frac{\Phi^{\prime}(x)}{16 k^{2}} \sin 4 k x .
$$

Use of the boundary conditions

$$
\psi(0)=\psi\left(\frac{\pi}{2}\right)=0
$$

and the Fourier series

$$
\Phi(x)=\sum_{n=1}^{\infty} a_{n} \cos 2 n x
$$

shows that

$$
\mu=\frac{a k}{2}
$$

so that

$$
\lambda=4 k^{2}+\frac{\epsilon a}{2 k} .
$$

Similarly for case 1.) we find

$$
\lambda=4 k^{2}-\frac{\epsilon a}{2}
$$

so that 


$$
\left|\lambda_{k+1}-\lambda_{k}\right|=\left|\epsilon a_{2 k}\right|
$$

It is evident from the previous development that Erdelyi's result is not correct in general, but Magnus has shown [4] that in an average sense it is correct. If

$$
\gamma_{1}<\gamma_{2}<\gamma_{3}<\cdots
$$

denote the $\lambda$ corresponding to even solutions of period $\pi$ and

$$
\sigma_{1}<\sigma_{2}<\sigma_{3}<\cdots
$$

correspond to odd solutions of period $\pi$. Magnus showed that

$$
\lambda_{0}+\sum_{n=1}^{\infty} \gamma_{n}-\sigma_{n}=-\sum_{n=1}^{\infty} a_{2 n} .
$$

A similar result holds for cases 3.) and 4.).

\section{A Special Case}

The previous results were all derived under the assumption that $\Phi(\mathrm{x})$ was differentiable. One can show that the asymptotic forns developed here do not hold when $\Phi(x)$ is not differentiable. Such an example was treated in detail in reference [5]. The equation considered there is

$$
y^{11}+\lambda^{2} Q(x) y=0
$$




$$
\begin{array}{rl}
Q(x)=1 & |x| \leq 1 \\
=a^{2} & 1<|x| \leq L
\end{array}
$$

and 1 t is shown there that

$$
\begin{aligned}
\lambda_{2 n} & =\left[\frac{2 n}{1+a(L-I)}\right] \frac{\pi}{2}+\theta \frac{\pi}{2} \\
\lambda_{2 n-1} & =\left[\frac{2 n}{1+a(L-1)}\right] \frac{\pi}{2}+\theta \frac{\pi}{2} \\
\lambda_{2 n}^{\prime} & =\left[\frac{2 n-1}{1+a(L-I)}\right] \frac{\pi}{2}+\theta \frac{\pi}{2} \\
\lambda_{2 n-1}^{\prime} & =\left[\frac{2 n-1}{1+a(L-1)}\right] \frac{\pi}{2}+\theta \frac{\pi}{2} .
\end{aligned}
$$

$[\mathrm{x}]$ denotes the greatest integer less than or equal to $\mathrm{x}$ and the $\theta$ 's must satisfy the inequality

$$
0 \leq \theta<1 \text {. }
$$

Here evidently

$$
\lambda_{k}=\frac{k \frac{\pi}{2}}{I+2(L-I)}+O(1)
$$

and the instability interrals are $O(1)$. 


\section{ACKANOWLEDGMENT}

The author wishes to thank Prof. W. Magnus for arousing his interest in this area of research, and for helpful suggestions made in the course of numerous discussions. 


\section{Bibliography}

[I] Borg, Göran

[2] Magnus W. and Shenitzer, A.

[3] Erdélyi, A.

[4] Magnus, W.

[5] Hochstadt, Harry
- Elne Umkehrung der Sturm-Ii ouv1lleschen Eigenwertaufgabe; Acta Math., 78, 1-96 (1946).

- Hill's Equation, Part I. General Theory; N.Y.U., Inst. Math. Sci., Div. EM Res., Research Report No. BR-22, 1957.

- Ueber die rechnerische Ermfttiung von Schwlngungsvorgängen etc.; Archiv der Elektrotechnik, 29, 473-489 (1935).

- The discriminant of Hill's equation; N.Y.U., Inst. Math. Sc1., Div. EM Res., Research Report No. BR-28, 1959.

- A special Hill's equation with discontinuous coefficients 3 N.Y.U., Inst. Math. Sci., Div. EM Res., Research Report No. BR-32, 1960. 


\section{A LABAMA}

Commender

Army Rocket and Guided Missile Agency

ATTN: ORDXR-OTI

Redstone Arsenal, A labama

\section{BELGIM}

Commander
European of fice, ARDC
47 Rue Cantersteen
Brussels, Belgiun

(3 Copies)

Brussels, Belgium

\section{CALIFORNIA}

Applied Mathematics and Statistics

Laboratory

Stanford University

St anford, Californt

Department of Mathematics

University of Californis

Eerkeley, Californla

Commander

Air Force Flight Test Center

ATT: Technical Library

Edwards Air Force Base, California

The Rand Corporation

(2 Coples)

Technical Library

1700 Main Street

Santa Monica, California

Commander

Ist Missile Division

ATIN: Operations Rnalysis office

Vandenburg Air Force Base, California

\section{CONNECT IC UT}

Department of Mathematics

Yale University

New Haven, Connecticut

\section{DISTRICT OF COLUMBIA}

Office of Naval Research

Department of the Navy

ATTN: Code 432

Washington 25, D.C.

\section{Director}

Department of Commerce

office of Technical Services

washington 25, D.C.

Administrator

(6 Copies)

National Aeronatics and

Space Administration

ATT: Documents Library

1520 H Streat, N.W.

Washingt on 25, D.C.

Library

National Bureau of Standards

Washington 25, D.C.

Data Processing Systems Division

Natlonal Bureau of Standards

ATTN: Mr. Rusgell A. Kirsch

Washington 25, D.C.

Applied Mathematics Division National Bureau of Standards Waghingt on 25, D.C.
Headquarters, USAF

Assistant for Operations Analysis

Deputy Chief of Staff, Operations, AFOOA

Washington 25, D.C.

Commander (2 Coples)

Air Force office of Scientific Research

ATTN: SRM

Washington 25 , D.C.

Director

U.S. Naval Research Laboratory

ATTN: Library

Washington 25, D.C.

Commander, AFRD

(2 Copies)

ATTN: Technical Library

Washington 25, D.C.

Nationsl Science Foundation

Program Director for Mathematical

Sciences

Washington 25, D.C.

Grnadian Jolnt Staff

ATTN: DRB/DSIS

2450 Massachusetts Avenue, N.W.

washington, D.C.

\section{ILLINOIS}

Department of Mathematics

Northwestern University

Evanston, Illinols

Laboratories for Applied Sciences

University of Chicago

Museum of Science and Industry

ATTN: Library, W-305

Chicago 37, Illinols

Department of Mathematics

University of Chicago

Chicago 37, Illinola

Department of Mathemstics

Untversity of Illinois

Urbana, Illinois

\section{INDIANA}

Department of Mathematics

Purdue Undversity

Lafayette, Indlana

\section{MARY LAND}

Institute for Fluid Dynamics and

Applied Mathematicg

Untversity of Marvland

College Park, Maryland

Mathematics and Physics Library

The John hopkins University

Baltimore, Maryland

Disector

National Security Agency

ATTN: Dr. H.H. Campelgn

Fort George G. Meade, Maryland

\section{MICHIGAN}

Department of Mathematics

Wayne State University

Detroit 1, Michigan
MINNESOTA

Department of Mathematics

Folwell Hall

Unt vergity of Minnesota

Minneapolis, Minnesota

Department of Mathematics

Institute of Technology

Engineering Building

University of Minnesots

Minneapolis, Minnesota

\section{MISSOURI}

Department of Mathematics

Washington University

St. Louis 8, Missouri

Department of Mathematics University of Missouri

Columbia, Missouri

\section{NEBRASKA}

Comma nder

Strategic A Ir Command

ATTN: Operations Analysis

offutt Air Force Base

Omahe, Nebraska

\section{NEW JERSEY}

The James Forrestal Research Center Library

Princeton University

Princeton, New Jersey

Library

Institute for Advanced Study

Princeton, New Jersey

Department of Mathematics

Fine $\mathrm{Hall}$

Princeton University

Princeton, New Jersey

Commanding Geners]

Signal Corps Engineering Laboratory

ATTN: SIGFM/ELRPO

Ft. Monmouth, New Jersey

\section{NEW MEXICO}

Comma nder

AIr Force Misslle Development Center

ATIN: Technicel Library, HDOI

Holloman Air Force Base, New Mexico

Commander

Air Force Special Weapons Center

ATTN: Techntcel Librar, SWOI

Kirtland Air Force Base

Al buque rque, New Mexdco

\section{NEW YORK}

Professor j. Wolfowit z

Mathematics Department

White $\mathrm{Ha} 11$

Cornell Und versity

Ithaca, New York

Department of Mathematics
Syracuse University

Syracuse, New York

Institute for Mathematical Sciences New York University

RTIN: Professor M. Kline

25 Waverly Place

New York, New York 
Institute for Aeronautical Sciences ATTN: Librarian

2 East tlith Street

New York If, New York

NORTH CAROLINA

Demartment of Mathematics

University of North Carolina

Chapel Hill, North Carolina

Department of Statistics

University of North Carolina

Chapel Hill, North Carolina

Office of Ordnance Research Box CM

(2 Copies)

Duke Station

Durham, North Carolina

Department of Mathematics

Duke University

Duke Station

Durham, North Carolina

\section{OHIO}

P. O. BOX AA

Wright- Patterson Air Force Base

Ohio

Commander

Wright Air Development Division

ATTN: WCOSI

Wrizht-Patterson A1r Force Rase

Ohio

Commander

Aeronautical Research Laboratories

ATTN: Technical Library

Wright-Patterson Air Force Base

Ohio

USAF Institute of Technology

Library

(2 Copies)

ATTN: MCLI-ITLIB

Building 125, Area $B$

Wrizht-Patterson Air Force Base

Ohio

\section{PENNSYLVANIA}

Department of Mathematics

Carnegie Institute of Technology

Pittsburgh, Pennsylvania

Department of Mathematics

University of Pennsylvania

Philadelphia, Pennsylvania

\section{TENNESSEE}

AEDC Library

ARO, Inc.

Arnold AF Station, Tennessee

U.S. Atomic Eneryy Comission Technical Information Service

Extension

P. 0 . Box 62

Dak Ridge, Tennessee

\section{TEXAS}

Applied Mechanics Reviews

Southwest Research Institute

(2 Copies)

8500 Culebra Poad

San Antonio 6 , Texas

Department of Mathematics

Rice Institute

Houston, Texas

\section{VIRGINIA}

Armed Services Technical

Information Agency (10 Copies)

ATTN: TIPDR

Arlington $\mathrm{Hall}$ Station

Arlington 12, Virginfa

WISCNNSIN

Department of Mathenatics

University of Wisconsin

Madison, Wisconsin

Nathematics Research Center,

U.S. A rmy

ATTN: R.E. Langer

University of Wisconsin

Madison, Wisconsin

\section{JUN 201961}

Date Due

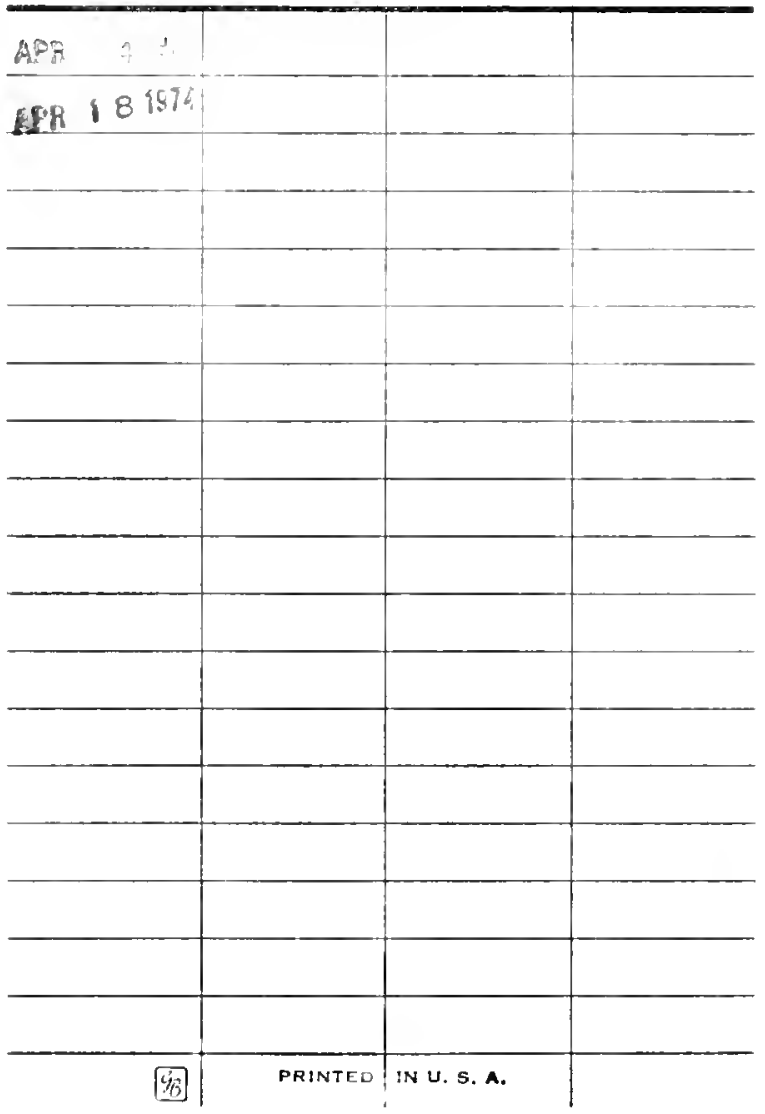


IITT

$\mathrm{BP}-36$ $c \cdot 2$

Hochstadt

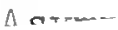

A atron

$\cdots$

... it

IYY

$\mathrm{BR}-36$

Hochstadt

AlI THUK

Asmptotic estimates for the

Sturn-diouville spectrum

c. 2

.29

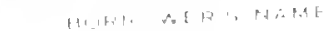

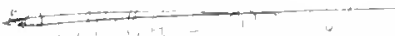

is

: $\quad 1-4 a_{4}+4$

N. Y. U. Institute of

Mathematical Sciences

25 Waverlyta

New York 3, N. Y.

4 Washinctin Place 
(n)

$\therefore$ ind 